\title{
EVALUATION OF MODIFIED CYTOSPIN SLIDE MICROSCOPY FOR DETECTION OF ACID FAST BACILLI IN BRONCHOALVEOLAR LAVAGE FLUID FOR DIAGNOSIS OF PULMONARY TUBERCULOSIS
}

\author{
Anam Imtiaz, Aamer Ikram*, Gohar Zaman**, Luqman Satti***, Fatima Sana** \\ Combined Military Hospital Lahore/National University of Medical Sciences (NUMS) Pakistan, *National Institute of Health, Islamabad Pakistan, **Armed Forces \\ Institute of Pathology/National University of Medical Sciences (NUMS) Rawalpindi Pakistan, ***Pakistan Naval Ship Shifa Hospital, Karachi Pakistan
}

\section{ABSTRACT}

Objective: To evaluate the cytospin slide microscopy method for detection of acid fast bacilli (AFB) in bronchoalveolar lavage (BAL) fluid comparing it with concentrated smear microscopy.

Study Design: Cross-sectional validation study.

Place and Duration of Study: Department of Microbiology, Armed Forces Institute of Pathology (AFIP), Rawalpindi, from Dec 2016 to Sep 2018.

Methodology: BAL specimens from suspected tuberculosis patients who were sputum smear negative, submitted to AFIP for diagnosis were included in the study. Smears for microscopy were prepared with the modified cytospin method as well as the standard concentrated technique. The prepared smears from both methods were stained with Ziehl-Neelsen (ZN) staining method and examined under $100 \times$ oil immersion lens. TB culture performed on BACTEC MGIT 960 automated culture system (Becton Dickinson, USA) was taken as gold standard for TB diagnosis. The two methods were compared in terms of sensitivity, specificity, positive predictive value and negative predictive value.

Results: Out of the 130 samples tested, 62 (47.7\%) were positive on culture using MGIT 960 system. The sensitivity, specificity, positive predictive value, negative predictive value and diagnostic accuracy of the modified cytospin method for pulmonary TB diagnosis was found to be $68.3 \%, 100 \%, 100 \%, 77 \%$ and $84.6 \%$, respectively.

Conclusion: The sensitivity of the modified cytopsin smear method was significantly higher than that of the concentrated method. The study concludes that this is a simpler and more accurate method for BAL fluid microscopy.

Keywords: Acid fast bacilli, Bronchoalveolar lavage, Cytospin, Microscopy, Pulmonary tuberculosis.

This is an Open Access article distributed under the terms of the Creative Commons Attribution License (https://creativecommons.org/licenses/by-nc/4.0/), which permits unrestricted use, distribution, and reproduction in any medium, provided the original work is properly cited.

\section{INTRODUCTION}

Tuberculosis (TB) remains a major public health issue particularly for the developing countries. It is a leading cause of death due to bacterial infection. Around 10 million new cases and 1.3 million deaths were reported from TB in the year 20171. No effective vaccine is available, so any effective control strategy has to focus on the early and efficient diagnosis and prompt treatment of the disease. Pakistan shares the 5 th highest burden of the disease ${ }^{2}$. Ours is a resource poor country and the economic burden due the disease is ever increasing. There is a dire need to focus on cost effective methods for its diagnosis.

The simplest, most widely used and cost effective method of diagnosis of active pulmonary TB is examination of stained sputum smear under the microscope. However, the sensitivity of this method varies with the availability of experienced staff, quality and nature of the specimen, smearing and staining technique and availability of a good microscope. Even under the best

Correspondence: Dr Anam Imtiaz, House No. 12, Lane 1, Sector-E, DHA Phase-II, Islamabad Pakistan

Received: 18 May 2019; revised received: 19 Jul 2019; accepted: 05 Aug 2019 conditions only approximately $50 \%$ cases are detected $^{3}$. Around $30-50 \%$ patients of pulmonary TB are sputum smear negative or sputum scarce ${ }^{4}$. Studies show that these patients with sputum smear-negative pulmonary TB are still able to transmit the disease to other people and contribute significantly to the disease burden $^{5}$. In these patients, fiber optic bronchoscopy (FOB) has been used to obtain bronchoalveolar lavage fluid (BAL) for analysis. Although, the diagnostic yield of BAL fluid culture for acid-fast bacilli (AFB) is high, but this method takes 2-6 weeks. In contrast, BAL fluid smear staining for AFB produces early results but with very low sensitivity since the threshold for detecting bacilli on simple light microscopy is about 5000 to 10000 bacilli per $\mathrm{ml}$ of the sample ${ }^{6-8}$. Several attempts have been made over the years to improve microscopy sensitivity by pre-processing samples with concentration methods including centrifugation, $\mathrm{N}$-acetyl Lcysteine $\mathrm{NaOH}$ and phenol-ammonium sulfate precipitation. Various modifications in staining have also been tried, like Kinyoun stain and fluorescent microscopy ${ }^{9}$. Cytospin slides have been reported to improve detection of AFB in cerebrospinal fluid (CSF) and BAL fluid samples. The technique is based on the rationale 
that the sample is concentrated over a small area on slide in the form of a smear and there is no loss of AFB in supernatant as happens in centrifugation ${ }^{10}$. Cytospin method has been used for Gram stained smears of fluid specimens where sensitivity is significantly improved with this method.

In this study, we will evaluate the cytospin technique for detection of AFB in BAL fluid comparing it with the concentrated smear microscopy. Not many studies have been done to evaluate this method of slide preparation for AFB detection.

The objective of the study was to evaluate the cytospin slide microscopy for detection of AFB in BAL fluid comparing it with already in use concentrated smear microscopy. Positive culture on MGIT 960 TB system will be considered as the gold standard for TB diagnosis.

\section{METHODOLOGY}

This was a comparative cross-sectional validation study carried out in the Department of Microbiology, Armed Forces Institute of Pathology (AFIP), Rawalpindi from December 2016 through September 2018. Permission was obtained from Institutional review board.

A total of 130 BAL fluid samples were included in the study through non probability convenience sampling. The sample size was calculated using the sensitivity and specificity sample size calculator, taking precision as 0.08 and $95 \%$ confidence interval. The values of sensitivity and specificity of cytospin microscopy method were taken from literature ${ }^{9-12}$. BAL fluid of suspected pulmonary TB cases, submitted to AFIP Rawalpindi for diagnosis were included in the study. Suspected cases were defined as productive cough for $>2$ weeks duration and/or radiographic findings (Chest X-ray or HRCT). Clinical samples of both genders, irrespective of age were included. Repeat samples and samples of patients taking antituberculosis treatment for $>2$ weeks were excluded from the study. All cases included were sputum smear negative defined as cases whom three consecutive early morning sputum samples did not reveal acid fast bacilli when examined by microscopy.

For concentrated smear method, BAL samples were pretreated using the Sodium hydroxide-Nacetyl-N-cysteine method for digestion and decontamination and centrifuged at $3000 \mathrm{rpm}$ for 20 minutes $^{11}$. The supernatant was discarded and the sediment was smeared on to a microscope slide over a $1 \times 2 \mathrm{~cm}$ area and stained by Ziehl-Neelsen (ZN) cold staining method.

For modified cytospin slide method, $0.5 \mathrm{ml}$ of BAL fluid was loaded onto cytospin slide chamber of Cytospin 4 cytocentrifuge machine (Thermo Fisher scientific, USA) and centrifuged at $1000 \times \mathrm{g}$ for 5 minutes. The slides were dried, fixed with $95 \%$ ethyl alcohol and stained.

The prepared smears from both methods were stained with $\mathrm{ZN}$ cold staining method. Slides were examined under $100 \times$ oil immersion lens, at least 300 fields were examined before reporting as negative. Incase AFB were seen, then bacillary load was established as; 1-2 AFB/300 fields characterized as doubtful, 1-9/100 fields as 1+, 1-9/10 fields $2+, 1-9 /$ field as $3+$ and $>9 \mathrm{AFB} /$ field as $4+$ (numerous) $^{14}$.

The modified cytospin slides were re-examined using a criteria of 50 fields/slide. All AFB slides from both methods were examined independently by two microbiology registrars.

TB culture was performed on BACTEC MGIT 960 automated culture system (Becton Dickinson, USA) ${ }^{13}$.

Data analysis was performed on SPSS version 21. For quantitative variables mean $( \pm S D)$ was calculated. Sensitivity, specificity, positive predictive value (PPV), negative predictive value (NPV) and diagnostic accuracy were calculated for the two methods keeping $\mathrm{TB}$ culture on MGIT 960 as the reference standard. The chi-sqaure test was used for categorical variables, $p$ value $<0.05$ was considered significant.

\section{RESULTS}

The mean age of the patients was $39.8(\mathrm{SD}=12.7)$ years. There were $105(80.8 \%)$ male patients while the rest were females (female to male ratio 1:4.2). Out of the total 130 samples included in the study $62(47.7 \%)$ were culture positive on MGIT 960 system, while 43 $(33.1 \%)$ and $23(17.7 \%)$ were positive on modified cytospin method and concentrated smear method, respectively. The $2 \times 2$ table for cytospin and concentrated method keeping TB culture as reference standard is presented below (table-I) and the sensitivity, specificity, positive predictive value, negative predictive value, and diagnostic accuracy for both methods of smear preparation (table-II). The sensitivity of cytospin method was significantly greater than the concentrated method for AFB microscopy $(p<0.001)$.

Microscopy using the cytospin method showed an increased density of bacilli per field. All the positive samples on cytospin smear microscopy were 
graded ' $4+$ '. Since the smear was of smaller area when made with cytospin method, less time was required to examine the whole smear area compared to $1 \times 2 \mathrm{~cm}$ smear made from the concentrated specimen. The results were the same when 50 fields were observed for the cytospin slides. method. BAL fluids were pretreated with $4 \%$ Sodium hydroxide $(\mathrm{NaOH})$ before loading onto cytospin chamber to prevent clogging of chambers by glutinous BAL samples. They also used poly-L-lysine coated slides to prevent loss of bacilli during staining since the proteins in BAL are denatured by $\mathrm{NaOH}$. We used

Table-I: $2 \times 2$ table for concentrated and cytospin method.

\begin{tabular}{|c|c|c|c|c|c|c|c|}
\hline & & \multicolumn{6}{|c|}{ TB Culture } \\
\hline \multirow{3}{*}{$\begin{array}{l}\text { Concentrated } \\
\text { Method }\end{array}$} & & Positive & Negative & \multirow{3}{*}{$\begin{array}{l}\text { Cytospin } \\
\text { Method }\end{array}$} & & Positive & Negative \\
\hline & Positive & $\mathrm{TP}=23$ & $\mathrm{FP}=0$ & & Positive & $\mathrm{TP}=43$ & $\mathrm{FP}=0$ \\
\hline & Negative & $\mathrm{FN}=40$ & $\mathrm{TN}=67$ & & Negative & $\mathrm{FN}=20$ & $\mathrm{TN}=67$ \\
\hline
\end{tabular}

$T P=$ True positive, $F P=$ False positive, $F N=$ False negative, $T N=$ True negative

Table-II: Compares the cytospin smear method with the concentrated method keeping TB culture as the gold standard for diagnosis.

\begin{tabular}{l|c|c|c|c|c}
\hline & $\begin{array}{c}\text { Sensitivity \% } \\
\mathbf{( 9 5 \% ~ C I ) ~}\end{array}$ & $\begin{array}{c}\text { Specificity \% } \\
\mathbf{( 9 5 \% ~ C I ) ~}\end{array}$ & $\begin{array}{c}\text { PPV \% } \\
\mathbf{( 9 5 \% ~ C I )}\end{array}$ & $\begin{array}{c}\text { NPV \% } \\
\mathbf{( 9 5 \% ~ C I ) ~}\end{array}$ & $\begin{array}{c}\text { Diagnostic Accuracy } \\
\mathbf{9} \text { (95\% CI) }\end{array}$ \\
\hline $\begin{array}{l}\text { Cytospin Smear } \\
\text { Microscopy }\end{array}$ & $68.3(55.3-79.4)$ & $100(100-100)$ & $100(100-100)$ & $77(70-82.8)$ & $84.6(77.2-90.3)$ \\
\hline $\begin{array}{l}\text { Concentrated } \\
\text { Smear Microscopy }\end{array}$ & $36.5(24.7-49.6)$ & $100(100-100)$ & $100(100-100)$ & $62.6(58.1-66.9)$ & $69.2(60.5-77)$ \\
\hline
\end{tabular}

$C I=$ Confidence Interval, $P P V=$ Positive predictive value, $N P V=$ Negative predictive value

\section{DISCUSSION}

A timely diagnosis and proper drug treatment can cure patients with the tuberculosis. Although TB culture is the gold standard for diagnosis but it takes 2-6 weeks ${ }^{15}$. Nucleic acid amplification based methods provide early diagnosis within hours, however they require equipment and expertise which are not widely available ${ }^{6}$. Microscopy of stained smears is the simplest method for diagnosis of tuberculosis. This is the only tool available in most resource poor diagnostic facilities. Improving the efficacy of this method may help in improving TB diagnosis.

Overall, $80.8 \%$ cases were males, a finding concurrent with other studies ${ }^{7,8}$.

TB culture on MGIT 960 was taken as the reference standard. The culture positivity was $47.7 \%$ in our study. Higher positivity rates have been reported in some regional studies ${ }^{6,7}$. While another study reports that MGIT culture was positive in $22 \%$ cases of clinically or radiologically suspected cases of pulmonary $\mathrm{TB}^{16}$.

The sensitivity of concentrated method in our study was $36.5 \%$. Similar results have been reported by other studies ${ }^{11,19}$.

In the present study the method of microscopy evaluated was adapted from Zheng et al study who evaluated the modified cytospin slide method on BAL fluid specimen for diagnosis of pulmonary TB. They reported a sensitivity of $91.5 \%$ with the modified the BAL samples directly without any prior treatment since there was no clogging during our pilot study. Also we used the cytospin settings of $1000 \mathrm{~g}$ for 5 minutes, different from that used by Zheng et al who used 250 for 5 minutes ${ }^{11}$. During the pilot study we compared both settings and found no difference in results. The authors in the present study favour the $1000 \mathrm{~g}$ settings as the smears seemed better formed with this speed. Chen et al used this setting for cytsopin of CSF samples for tuberculous meningitis diagnosis and reported and improved sensitivity of $93.8 \%{ }^{10}$. Other similar studies have also reported improved sensitivity of cytospin smears for CSF 17,18 . The results of our study showed a sensitivity of $68.3 \%$, $100 \%$ specificity and an overall diagnostic accuracy of $84.6 \%$.

The cytospin method significantly improves the AFB detection in fluid samples compared to the concentrated method. The bacilli are easier to detect as they are concentrated on a small area on the slide. The number of bacilli per field is also higher than that with other methods. The modified cytospin slides are also less time consuming and safer since there is minimal handling of the specimen and does not require any pretreatment. Also, the AFB can be accurately observed on microscopy in less time, so a less experienced microscopist may be able to accurately report these slides. Further multicentric studies with larger sample sizes are required for further validation of the method for pulmonary TB diagnosis. 


\section{CONCLUSION}

The modified cytospin slide microscopy for diagnosis of pulmonary TB is a simple, easy to perform technique that significantly improves the sensitivity of BAL fluid microscopy compared to the concentrated smear method.

\section{CONFLICT OF INTEREST}

This study has no conflict of interest to be declared by any author.

\section{REFERENCES}

1. World Health Organization. WHO Global tuberculosis report 2018. Geneva, Switzerland: WHO; 2018. [Internet]. Available from:https://www.who.int/tb/publications/global_report/ en/ [Assessed at: Feb 2, 2019]

2. World Health Organization EMRO. Tuberculosis, Programmes Pakistan. [internet] Geneva: World Health Organization; 2018. [cited 2019 July 2] Available from: http://www.emro.who.int/ pak/programmes/stop-tuberculosis.html

3. Campos LC, Rocha MVV, Willers DMC, Silva DR. Characteristics of patients with smear-negative pulmonary tuberculosis (TB) in a region with high TB and HIV prevalence. PLoS ONE 2016; 11(1): e0147933.

4. Nikbakhsh N, Bayani M, Siadati S. The value of bronchoalveolar lavage in the diagnosis of sputum smear-negative Pulmonary Tuberculosis. Iran J Pathol 2015; 10(1): 35-40.

5. Behr MA, Warren SA, Salamon H, Hopewell PC, Ponce de Leon A, Daley CL, et al. Transmission of mycobacterium tuberculosis from patients smear-negative for acid-fast bacilli. Lancet 1999; 353: 444-49.

6. Khalil KF, Butt T. Diagnostic yield of bronchoalveolar lavage gene Xpert ${ }^{\circledR}$ in smear-negative and sputum-scarce pulmonary tuberculosis. J Coll Physicians Surg Pak 2015; 25(2): 115-18.

7. Kalawat U, Sharma KK, Reddy PNR, Kumar AG. Study of bronchoalveolar lavage in clinically and radiologically suspected cases of pulmonary tuberculosis. Lung India 2010; 27(3): 122-24.

8. Ahmad M, Ibrahim WH, Sarafandi SA, Shahzada KS, Ahmed S, Haq IU, et al. Diagnostic value of bronchoalveolar lavage in the subset of patients with negative sputum/smear and mycobacterial culture and a suspicion of pulmonary tuberculosis. Int J Infect Dis 2019; 82: 96-10.
9. Singhal R, Myneedu VP. Microscopy as a diagnostic tool in pulmonary tuberculosis. Int J Mycobacteriol 2015; 4(1): 1-6.

10. Chen P, Shi M, Feng GD, Liu JY, Wang BJ, Shi XD, et al. A highly efficient Ziehl-Neelsen stain: Identifying de novo intracellular Mycobacterium tuberculosis and improving detection of extracellular M. tuberculosis in cerebrospinal Fluid. J Clin Microbiol 2012; 50(4): 1166-70.

11. Zheng LH, Jia HY, Liu XJ, Sun HS, Du FJ, Pan LP, et al. Modified cytospin slide microscopy method for rapid diagnosis of smearnegative pulmonary tuberculosis. Int J Tuberc Lung Dis 2016; 20(4): 456-61.

12. Shanholtzer CJ, Schaper PJ, Peterson L. Concentrated gram stain smears prepared with a Cytospin centrifuge. J Clin Microbiol 1982; 16(6): 1052-56.

13. Siddiqi SH, Rusch-Gerdes S. MGIT procedure manual. Geneva, Switzerland: Foundation for Innovative New Diagnostics (FIND); 2006

14. World Health Organization. Laboratory service in tuberculosis control Part II: Microscopy. Geneva, Switzerland: WHO; 1988 Available from: https://apps.who.int/iris/bitstream/handle/ 10665/65942/WHO_TB_98.258_(part2).pdf;jsessionid=776BA4A 1BB33A3AA2FC6C0B1E8D6C9F2? sequence $=2$

15. Tortoli E, Cichero P, Piersimoni C, Simonetti MT, Gesu G, Nista D. Use of BACTEC MGIT 960 for recovery of Mycobacteria from clinical specimens: multicenter study. J ClinMicrobiol 1999; 37(11): 3578-82.

16. Revendran J, Nair G, Uppe A, Sengal V, Sinha K, Jain S. Smear negative pulmonary tuberculosis: clinico-radiological profile and diagnostic role of bronchoscopy with bal studies in indian population. Eur Respir J 2017; 50(61): PA2711.

17. Feng GD, Shi M, Ma L, Chen P, Wang BJ, Zhang M, et al. Diagnostic accuracy of intracellular mycobacterium tuberculosis detection for tuberculous meningitis. Am J Respir Crit Care Med 2014; 189(4): 475-81.

18. Heemskerka AD, Donovana J, Thu DDA, Marais S, Chaidir L, Dung VTM, et al. Improving the microbiological diagnosis of tuberculous meningitis: A prospective, international, multicentre comparison of conventional and modified Ziehl-Neelsen stain, GeneXpert, and culture of cerebrospinal fluid. J Infect 2018; 77: 509-15.

19. Gowda NC, Ray A, Soneja M. Evaluation of Xpert® Mycobacterium tuberculosis/rifampin in sputum-smear negative and sputum-scarce patients with pulmonary tuberculosis using bronchoalveolar lavage fluid. Lung India 2018; 35: 295-300. 